\title{
Niveles de eficiencia en las clínicas estomatológicas en Matanzas, Cuba
}

\section{The efficiency of the public dental care services in Matanzas, Cuba}

\author{
Magalys Chaviano Moreno'; Zoe Sánchez Delgado"; Anaí García Fariñas'"' \\ 'Máster en Economía de la salud. Dirección Provincial de Salud de Matanzas. \\ Matanzas, Cuba. \\ "Máster en Economía de la salud. Asistente. Dirección Provincial de Salud de \\ Matanzas. Matanzas, Cuba. \\ "I'Máster en Economía de la Salud. Investigadora Agregada. Instituto de Farmacia y \\ Alimentos. Universidad de La Habana. La Habana, Cuba.
}

\section{RESUMEN}

Introducción El estudio de eficiencia en unidades de salud contribuye a diseñar estrategias de distribución de gastos hacia alternativas que lleven a alcanzar mejores resultados con los escasos recursos disponibles.

Objetivos Conocer el nivel de eficiencia en las clínicas estomatológicas en Matanzas, identificar las de mejor práctica y puntualizar las áreas potenciales de mejora para cada unidad.

Métodos Se realizó una investigación descriptiva retrospectiva en las 10 clínicas estomatológicas en la provincia Matanzas, Cuba, en el año 2006. Se emplearon tres indicadores de resultados y dos de recursos. Se utilizó el análisis envolvente de datos bajo el modelo de maximización de resultados y el supuesto de rendimientos constantes a escala.

Resultados El valor medio de eficiencia fue $90,07 \pm 18,3$. Seis unidades ( $60 \%$ ) resultaron eficientes y cuatro $(40 \%)$ fueron ineficientes. Se identificó como unidad de mejor práctica durante el 2006 a la clínica "César Escalante". La principal área de mejora fue el incremento del porcentaje de pacientes que no requirieron tratamiento, así como la disminución del gasto en medicamentos y materiales. Conclusiones Las clínicas estomatológicas en la provincia de Matanzas, en su mayoría, lograron en 2006 altos niveles de eficiencia. La información generada en 
su conjunto resulta de utilidad para la búsqueda de la excelencia en estas instituciones en tanto puede devenir en una herramienta para una mejor gestión.

Palabras clave: Eficiencia, análisis envolvente de datos, clínicas estomatológicas, Matanzas.

\section{ABSTRACT}

Introduction The study of efficiency in health units will contribute to designing strategies of distribution of expenses toward more efficient alternatives with the present available resources.

Objectives To determine the efficiency rate of public dental care services in Matanzas, to identify those units with the best practice and to ascertain the potential areas to be improved in each unit.

Methods A retrospective descriptive research study was made in the 10 dental clinics of the province in 2006. Three outcome indicators and two resource indicators were used. Also, data envelopment analysis technique under the outcome maximization model and the assumption of constant returns scale to scale were employed.

Results The mean value of efficiency was $90.07 \pm 18.3$. Six health units (60\%) were efficient whereas four (40\%) were inefficient. "Cesar Escalante" dental clinics was the best practice unit in 2006 . The principal area of improvement was the increase of percentage of patients that did not require treatment, as well as the decrease of drug and material expenses.

Conclusions Most of dental clinics in Matanzas exhibited high efficiency levels in 2006. The collected information is useful for the search of excellence in service and may also become a tool for better management.

Key words: Efficiency, data envelopment analysis, dental clinics, Matanzas.

\section{NTRODUCCI ÓN}

Con el fin de reducir las disparidades en salud que existen entre los países, numerosos acuerdos se han firmado por la comunidad mundial en relación con el derecho a la salud, así como sobre la responsabilidad ineludible de los gobiernos de diseñar políticas que aseguren el acceso a los servicios de salud para la totalidad de la población y que eliminen las iniquidades a un costo sostenible para la sociedad. ${ }^{1}$

El logro de sistemas de salud eficientes constituye un tema pendiente tanto para los estados como para las comunidades. Aún cuando fue este uno de los elementos más mencionados en el marco de las reformas de salud en Latinoamérica, todavía hoy se inserta frecuentemente en las agendas de discusiones económicas, políticas y de carácter social. 
La eficiencia según Palmer y otros, ${ }^{2}$ se define como la relación entre los resultados obtenidos en una actividad dada y los recursos utilizados, y donde los objetivos fijados se alcanzan sin desperdiciar los recursos. Pueden describirse, entonces, dos referencias para mejorarla; por una parte los beneficios no alcanzados y por la otra los recursos mal aprovechados.

Cuando se habla de eficiencia en salud, se hace referencia a alcanzar los mejores resultados con los escasos recursos disponibles para lo cual deben conocerse las formas y los procesos técnicos necesarios para lograrla. La Organización Panamericana de la Salud ha planteado ${ }^{3}$ que un sistema de salud es eficiente cuando es capaz de brindar un producto sanitario aceptable para la sociedad con un uso mínimo de recursos.

En especial, garantizar los servicios estomatológicos, incluso los básicos, deviene en un reto tanto para los prestadores de servicios como para las sociedades, pues esta actividad está acompañada de altos $\operatorname{costos}^{4-8}$ dados, entre otros elementos, por los numerosos recursos necesarios, la complejidad de los tratamientos, los requerimientos en términos de recursos humanos, equipamientos, condiciones de electricidad, agua e iluminación. El alto costo de la atención estomatológica se inserta como un elemento más que reafirma la necesidad de un uso eficiente de los recursos destinados a prestar estos servicios de salud. Cada vez se encuentran más estudios que intentan contribuir a un mejor proceso de toma de decisiones a partir del estudio de la eficiencia, tanto para acciones específicas como para las entidades que brindan estos servicios de salud. Ejemplo de ello son las investigaciones realizadas en Inglaterra, Noruega, Finlandia y Grecia. ${ }^{9-12}$ La región latinoamericana, no cuenta con grandes experiencias al respecto, aún cuando los gobiernos demandan cada vez más evidencias científicas que apoyen la extensión de los servicios en número y tipo.

En Cuba, como parte indisoluble del Sistema Nacional de Salud (SNS) se encuentra la atención estomatológica, ${ }^{13}$ a la cual luego del triunfo de la Revolución se han destinado numerosos recursos. Luego de pasado los peores momentos de la crisis económica de los años noventa del Siglo xx, hoy día, la atención estomatológica se encuentra en franco perfeccionamiento a nivel nacional. Los últimos cinco años se han caracterizado por un amplio proceso inversionista acompañado de una estrategia de descentralización en la formación de los recursos humanos.

Si bien en Cuba, no existen limitaciones de tipo financiero en el acceso a los servicios de salud y no media el pago directo por parte de los usuarios, esto no implica que se dejen de utilizar los recursos en su mejor uso alternativo, ${ }^{14}$ de aquí que el reto que hoy enfrenta el país en especial el área estomatológica está en lograr que la inversión realizada se traduzca en la mejoría de la salud bucal de la población así como en la satisfacción de las demandas de la población.

Matanzas es la más central de las provincias occidentales, cuenta con 14 municipios y en 8 de ellos (Matanzas, Varadero, Colón, Perico, Limonar, Jovellanos, Pedro Betancourt y Jagüey) se ubican las 10 clínicas estomatológicas de la provincia. Estas unidades tienen la misión de garantizar la atención estomatológica integral mediante acciones de promoción, prevención curación y rehabilitación, que contribuyan al mejoramiento de la salud bucal de la población. Esta provincia vive un escenario similar al nacional en lo referente a la recuperación económica, en especial en los servicios estomatológicos, sin embargo, no se conoce cuales son los niveles de eficiencia alcanzado por las clínicas que brindan estos servicios. Tampoco existen experiencias anteriores en el país respecto al tema de la eficiencia en las entidades que prestan servicios estomatológicos. Poder determinar cuáles son los 
niveles de eficiencia alcanzado por cada clínica, así como el efecto que determinadas variables contextuales tienen sobre la eficiencia en estas unidades contribuirá al perfeccionamiento de la gestión pública.

La presente investigación se desarrolló para responder a los siguientes objetivos: conocer el nivel de eficiencia de cada clínica estomatológica de Matanzas en el año 2006, identificar las unidades de mejor práctica en ese periodo, identificar las potencialidades para mejorar la eficiencia en cada clínica ineficiente detectada y evaluar el efecto que sobre la eficiencia tiene la condición de docente o no de la clínica (presencia de alumnos de la carrera de Estomatología de cuarto y quinto año) así como de la proporción de conjuntos dentales activos.

\section{MÉTODOS}

Se realizó una investigación descriptiva. Se eligió como horizonte temporal el año 2006. El universo de estudio fueron las 10 clínicas estomatológicas en la provincia Matanzas. Para determinar el nivel de eficiencia de las clínicas se procedió a la selección de indicadores de resultados y de recursos. Para la identificación de los indicadores de resultados se realizó una revisión bibliográfica y una consulta a expertos entre enero y marzo de 2007. La revisión bibliográfica incluyó el Programa Nacional de Estomatología, ${ }^{5}$ Programa de Calidad y Satisfacción en Estomatología, ${ }^{6}$ y documentos de la Organización Mundial de la Salud, así como de investigadores de prestigio en el tema. ${ }^{15-17}$ El grupo de expertos consultado estuvo compuesto por 17 profesionales vinculados a la atención estomatológica desde el nivel nacional hasta las propias clínicas, entre ellos epidemiólogos, economistas de la salud, administradores de salud y estomatólogos generales integrales. Se emplearon como criterios de inclusión: tener al menos 10 años de experiencia de trabajo. Una vez identificados los indicadores de resultados el equipo de investigación procedió a la selección de los posibles indicadores de recursos. La literatura sobre los requerimientos del análisis envolvente de datos ${ }^{18}$ establece que estos indicadores deben haber sido reconocidos por los que toman decisiones en las unidades a evaluar como elementos claves para alcanzar los resultados ya definidos.

Se empleó como modelo de optimización el de maximización de resultados debido a que internacionalmente se reconoce como adecuado ${ }^{19}$ cuando los directivos están interesados en un aumento de la productividad sin que necesariamente esto conlleve a una reducción de los recursos en uso, lo cual se aviene con la política de gobierno cubana en lo que a servicios de salud respecta. En relación con los rendimientos según la escala para este estudio se asumieron Rendimientos Constates a Escala (CRS, por sus siglas en ingles). El elemento fundamental que justificó esta decisión fue la existencia de publicaciones previas que señalan que en un grupo homogéneo de unidades es muy probable encontrarse en este caso. ${ }^{20} \mathrm{La}$ homogeneidad del grupo de unidades a evaluar en este estudio estuvo dada por su inserción en un sistema de salud único que tiene las características organizativas de asignación de recursos y de funcionamiento de las unidades, similares para todo el país.

Se calculó el índice de eficiencia productiva de cada clínica mediante la resolución de la función objetivo propuesta por Cooper y otros. ${ }^{21}$ Para la resolución automática de este modelo se empleó el Frontier Analyst Versión DEMO. Los niveles de eficiencia se presentaron en frecuencias absolutas para cada unidad. Se calculó el valor medio de ineficiencia. Además se evaluaron posibles diferenciales del índice de eficiencia según la condición de docente o no de la clínica de y la proporción de 
conjuntos dentales activos (número de unidades dentales y sillones que funcionaron durante del año / número de unidades dentales, sillones, asignados a la unidad). Para la comparación de los niveles de eficiencia entre los grupos conformados con cada variable se aplicó la técnica no paramétrica de comparación de rangos de Mann-Whitney. Se empleó programa de cálculo estadístico computarizado.

La unidad de mejor práctica se eligió de entre las eficientes a partir del porcentaje de empleo de cada clínica eficiente como referencia para el resto de las unidades. Las áreas potenciales de mejora para las clínicas ineficientes se exploraron tanto para los indicadores de resultados como para los indicadores de recursos, es decir se determinó el porcentaje en que deberían incrementarse los resultados y/o disminuirse los recursos para que las clínicas ineficientes alcanzaran los valores de las unidades eficientes.

\section{RESULTADOS}

La Dirección Nacional de Estomatología, del Ministerio de Salud Pública de Cuba $(\text { MINSAP })^{13}$ tiene establecido cinco indicadores para evaluar el resultado de las clínicas estomatológicas del país. Estos exploran la estabilidad en las acciones de promoción y prevención, la efectividad en los tratamientos necesarios para que la población en edades avanzadas logre conservar 20 ó más dientes funcionales y las acciones de rehabilitación (recuadro). Debido a que sólo los servicios de estomatología general básica fueron comunes a todas las clínicas se seleccionaron, para este estudio, los tres indicadores correspondientes a estos servicios: cobertura asistencial, porcentaje de la población que en el momento de ser examinada no requerían tratamiento estomatológico y porcentaje de altas de control de placa dentobacteriana.

Recuadro. Propuesta de indicadores de resultados de la clínica

\begin{tabular}{|c|c|c|}
\hline Nombre del indicador & Descripción del indicador & Área de resultados que evalúa \\
\hline $\begin{array}{l}\text { Porcentaje de la población que no requiere } \\
\text { tratamiento }\end{array}$ & $\begin{array}{l}\text { Número de pacientes que al examinarse } \\
\text { no requieren atención estomatológica / } \\
\text { total de pacientes examinados por cien }\end{array}$ & $\begin{array}{l}\text { Estabilidad en las acciones de } \\
\text { promodón y prevención así como en la } \\
\text { garantía de atención estomatológica } \\
\text { básica. }\end{array}$ \\
\hline Cobertura asistencial & $\begin{array}{l}\text { Es el producto de dividir el resultado de la } \\
\text { suma de los que no requieren } \\
\text { tratamiento más los tratamientos } \\
\text { concluidos entre el universo de población } \\
\text { por cien }\end{array}$ & $\begin{array}{l}\text { Incentiva el examen y el tratamiento no } \\
\text { sólo aurativo, sino también el } \\
\text { preventivo. }\end{array}$ \\
\hline $\begin{array}{l}\text { Porcentaje de controles de placa } \\
\text { dentobacteriana realizados }\end{array}$ & $\begin{array}{l}\text { Se calcula la totalidad de los controles de } \\
\text { placa dentobacteriana concluidos en } \\
\text { todos los gnupos/ los universos por cien. }\end{array}$ & $\begin{array}{l}\text { Este indicador evalúa las acciones } \\
\text { preventivas realizadas sobre el } \\
\text { principal factor de riesgo de las } \\
\text { enfermedades buco dentales, la higiene } \\
\text { bucal y el control de la placa dento } \\
\text { bacteriana, agente causal de las } \\
\text { mismas. }\end{array}$ \\
\hline Efectividad en Estomatología General Integral & $\begin{array}{l}\text { Para caloularlo se suman las obturaciones } \\
\text { realizadas con los tratamientos pulpo } \\
\text { radiculares y se le restaran las } \\
\text { extracciones dentarías }\end{array}$ & $\begin{array}{l}\text { Evalúa la efectividad en los } \\
\text { tratamientos conservadores, necesarios } \\
\text { para que la población en edades } \\
\text { avanzadas logre conservar } 20 \text { ó más } \\
\text { dientes funcionales. }\end{array}$ \\
\hline Aprovechamiento de los servicios de prótesis. & $\begin{array}{l}\text { Se suman los pacientes que han } \\
\text { concluido el tratamiento de prótesis, con } \\
\text { el número de aparatos protésicos de } \\
\text { cromo oobalto y de cerámica instalados y } \\
\text { se divide entre el número de especialistas } \\
\text { de prótesis que laboran. }\end{array}$ & $\begin{array}{l}\text { Evalúa las acciones de rehabilitación } \\
\text { realizadas. }\end{array}$ \\
\hline
\end{tabular}

Fuente: Programa Nacional de Estomatología Integral a la. Población. Cuba, 2006. 
En relación con los recursos materiales relevantes para la consecución de los resultados la Dirección Nacional de Estomatología considera que de los 1500 renglones de material gastable reconocidos internacionalmente como necesarios para los diferentes servicios que se brindan en una unidad de atención estomatológica, 40 son esenciales o fundamentales. De ellos, se tomaron los 25 relacionados directamente con los servicios básicos de estomatología. Se calculó el gasto por material gastable para el año 2006 a partir de la cantidad empleada de cada material y el precio oficial para Cuba. La medición de los recursos humanos implicados en el logro de los resultados se calculó a través del salario devengado durante el año por el equipo básico de salud bucal (estomatólogo y técnico de atención estomatológica) vinculado a los servicios de estomatología general. Tanto el costo por materiales como por recursos humanos se expresó en pesos cubanos del 2006.

Los valores medios y la distribución estándar de las variables de resultados y de recursos seleccionadas se muestran en la tabla 1 . La media del indicador "porcentaje de la población que no requiere tratamiento", fue de 55,27 \%, valor que pudiera interpretarse como adecuado, ya que más de la mitad de los pacientes que se examinaron en las clínicas no necesitaba atención estomatológica, o bien por no estar afectados o por mantener estable su salud bucal; sin embargo, la elevada dispersión encontrada entre las unidades señala que no deben hacerse conclusiones sólo en base al valor promedio. Los valores medios de "control de placa dentobacteriana" $(46,87 \%)$ encontrados indica que es necesario intensificar el accionar sobre los factores de riesgo de enfermedades bucales. La "cobertura asistencial" con un valor medio $(29,01 \%)$ puede considerarse como deficiente, con unidades que no lograron siquiera el $5 \%$ de cobertura.

Tabla 1. Distribución de los indicadores de recursos y de resultados. Matanzas 2006

\begin{tabular}{|c|c|c|c|c|c|}
\hline & \multicolumn{3}{|c|}{ Indicadores de resultados } & \multicolumn{2}{|c|}{ Indicadores de recursos } \\
\hline & $\begin{array}{l}\text { No requiere } \\
\text { tratamiento } \\
\text { (\%) }\end{array}$ & $\begin{array}{l}\text { Altas de control } \\
\text { dentobacteriana (\%) }\end{array}$ & $\begin{array}{l}\text { Cobertura } \\
\text { asistencial } \\
(\%)\end{array}$ & $\begin{array}{l}\text { Costo salario } \\
\text { (miles de pesos } \\
\text { cubanos) }\end{array}$ & $\begin{array}{l}\text { Costo materiales } \\
\text { (miles de pesos } \\
\text { cubanos) }\end{array}$ \\
\hline Media & 55,27 & 46,87 & 29,01 & 12,22 & 4,05 \\
\hline DS & 25,98 & 27,07 & 24,10 & 4,90 & 1,69 \\
\hline Mínimo & 5,38 & 18,70 & 3,81 & 14,98 & 2,10 \\
\hline Máximo & 90,66 & 99,08 & 92,23 & 23,62 & 7,50 \\
\hline
\end{tabular}

Los índices de eficiencia encontrados para cada clínica así como su ubicación municipal se muestran en la tabla 2. De las 10 unidades analizadas 6 resultaron eficientes para el $60 \%$. La distribución por municipios se comportó de la siguiente manera: Varadero, Jovellanos, Limonar y Pedro Betancourt, fueron los municipios donde todas las unidades fueron eficientes; en los municipios Matanzas y Jagüey, una de dos unidades analizadas resultó eficiente; en Colón se encontró la unidad que más bajo índice de eficiencia mostró durante el año. 
Tabla 2. Índice de eficiencia en clínicas estomatológicas según municipios, Matanzas, Cuba, 2006

\begin{tabular}{|c|c|c|}
\hline Clínica Estomatológica & $\begin{array}{c}\text { Rango de eficiencia } \\
\text { (\%) CRS }\end{array}$ & Municipio \\
\hline 27 de Noviembre & 39,25 & Colón \\
\hline Agramonte & 100,00 & Jagüey \\
\hline César Escalante & 100,00 & Matanzas \\
\hline Hermanos Almeida & 100,00 & Jovellanos \\
\hline Jagüey & 90,78 & Jagüey \\
\hline Leonilda Soumbill & 100,00 & Varadero \\
\hline Limonar & 100,00 & Limonar \\
\hline Pedro Betancourt & 100,00 & Pedro Betancourt \\
\hline Tercer Congreso & 83,55 & Matanzas \\
\hline XX Aniversario del Moncada & 87,10 & Perico \\
\hline
\end{tabular}

Los niveles de eficiencia en las clínicas no docentes fueron ligeramente superiores a las docentes. Aunque estas diferencias no fueron estadísticamente significativas $(p=0,055)$ la proximidad al punto de corte y el bajo número de unidades evaluadas pueden apuntar hacia la necesidad de explorar esta relación en una muestra mayor. En relación con la proporción de conjuntos dentales activos, los resultados no mostraron diferencias significativas entre los valores de eficiencia del grupo de unidades con el $100 \%$ de conjuntos activos y los que al menos tuvieron un conjunto deshabilitado durante el año.

En el análisis de cuáles unidades podían ser consideradas como las de mejor práctica se obtuvieron los siguientes resultados: la clínica "César Escalante" fue empleada como referencia para las 4 unidades ineficientes, la clínica "Hermanos Almeida" no fue referencia para ninguna unidad ineficiente y las restantes cuatro clínicas eficientes sirvieron de referencia sólo a una unidad ineficiente. De acuerdo a estos resultados se identificó como unidad de mejor práctica durante el 2006 a la clínica "César Escalante."

La figura muestra las áreas de mejoras potenciales tanto para indicadores de resultados como de recursos. Esta información permite identificar en cuáles variables deben concentrarse las acciones de los directivos para lograr mayores índices de eficiencia. Las cuatro clínicas ineficientes cuentan con posibilidades de mejorar su eficiencia si mejoran los resultados en los tres indicadores de resultados evaluados en esta investigación, sin embargo, especial atención debe darse al incremento de las personas que no requieren tratamiento. De los dos indicadores de recursos, analizados se encontró que en las clínicas estomatológicas ineficientes existe un consumo relativamente mayor de medicamentos los cuales en teoría pueden ser reducidos en el $2,52 \%$. La tabla 3 muestra los resultados específicos para cada unidad ineficiente. 


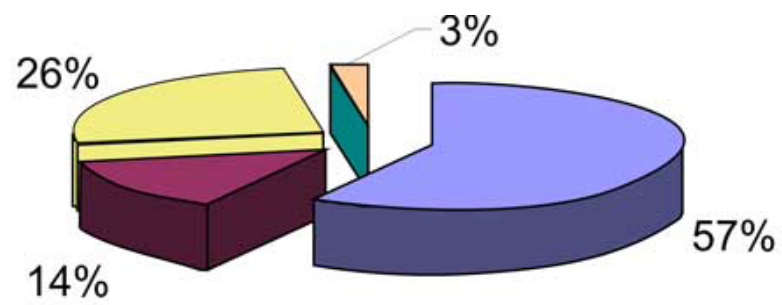

\begin{tabular}{l} 
पNRT $\begin{array}{l}\text { 口CPDB } \\
\square \mathrm{GM}\end{array}$ \\
\begin{tabular}{|l|c|c|}
\hline Variable & Leyenda & $\begin{array}{c}\text { \% de contribución a la } \\
\text { mejoría de la eficiencia }\end{array}$ \\
\hline No requiere tratamiento & NRT & 57 \\
Control de placa dento bacteriana & CPDB & 14 \\
Cobertura & C & 26 \\
Gasto en medicamento & GM & 3 \\
\hline
\end{tabular} \\
\hline
\end{tabular}

Fig. 1. Distribución de las áreas susceptibles de mejoras, clínicas estomatológicas Matanzas 2006.

\begin{tabular}{|c|c|c|c|c|c|c|c|c|c|c|}
\hline \multirow[t]{3}{*}{ Clínicas } & \multicolumn{6}{|c|}{ Indicadores de resultados } & \multicolumn{4}{|c|}{ Indicadores de recursos } \\
\hline & \multicolumn{2}{|c|}{$\begin{array}{l}\text { No requieren } \\
\text { tratamiento }\end{array}$} & \multicolumn{2}{|c|}{$\begin{array}{l}\text { Control de placa } \\
\text { dentobaceriana }\end{array}$} & \multicolumn{2}{|l|}{ Cobertura } & \multicolumn{2}{|c|}{ Gasto en salario } & \multicolumn{2}{|c|}{$\begin{array}{l}\text { Gasto en } \\
\text { medicamento }\end{array}$} \\
\hline & $\begin{array}{l}\text { Valor a } \\
\text { alcanzar }\end{array}$ & $\begin{array}{l}\% \text { de } \\
\text { incremento }\end{array}$ & $\begin{array}{l}\text { Valor a } \\
\text { alcanzar }\end{array}$ & $\begin{array}{l}\% \text { de } \\
\text { incremento }\end{array}$ & $\begin{array}{l}\text { Valor a } \\
\text { alcanzar }\end{array}$ & $\begin{array}{l}\% \text { de } \\
\text { incremento }\end{array}$ & $\begin{array}{l}\text { Valor a } \\
\text { alcanzar }\end{array}$ & $\begin{array}{l}\text { \% de } \\
\text { disminución }\end{array}$ & $\begin{array}{l}\text { Valor a } \\
\text { alcanzar }\end{array}$ & $\begin{array}{l}\text { 96 de } \\
\text { disminución }\end{array}$ \\
\hline $\begin{array}{l}27 \text { de } \\
\text { Noviembre }\end{array}$ & 72,76 & 154,8 & 47,64 & 154,8 & 51,46 & 154,8 & 14,36 & 0 & 6,82 & $-9,1$ \\
\hline Jagüey & 34,54 & 542,5 & 42,16 & 10,2 & 10,33 & 171,3 & 4,98 & 0 & 1,94 & $-22,3$ \\
\hline $\begin{array}{l}\text { Tercer } \\
\text { Congreso }\end{array}$ & 71,52 & 119,9 & 87,29 & 19,7 & 21,38 & 20,0 & 10,32 & 0 & 4,01 & $-4,9$ \\
\hline $\begin{array}{l}X X \\
\text { Aniversario } \\
\text { del } \\
\text { Moncada, }\end{array}$ & 72,99 & 14,8 & 41,09 & 14,8 & 22,11 & 28,2 & 12,14 & 0 & 2,85 & 0,0 \\
\hline
\end{tabular}

\section{DISCUSIÓN}

Un aspecto fundamental en la evaluación de la gestión de las organizaciones sanitarias es la capacidad de identificar y separar aquellas organizaciones, que de acuerdo con algún referente, gestionan correctamente de aquellas que lo hacen mal o por debajo de sus posibilidades. El presente trabajo caracterizó la eficiencia productiva de las clínicas estomatológicas en la provincia Matanzas, y aún cuando no se identificaron variables contextuales relacionadas con aspectos socio económicos, demográficos, gerenciales y de calidad, ${ }^{12,22}$ registradas como variables explicativas del comportamiento de la eficiencia, la información generada resulta de utilidad para la búsqueda de la excelencia en estas instituciones en tanto puede devenir en una herramienta para su mejor gestión.

En los últimos 10 años, puede observarse en la literatura un incremento discreto de publicaciones de estudios que evalúan la eficiencia en clínicas estomatológicas a partir del empleo de técnicas no paramétricas como el análisis envolvente de datos. Se destacan entre los países que han desarrollado estos estudios Noruega, Inglaterra y Finlandia. ${ }^{5} \mathrm{Si}$ bien los indicadores empleados en este tipo de estudio, 
para describir los recursos, son bastante similares a los utilizados en este, no resulta así para los de resultados pues algunos autores emplean indicadores de proceso como el número de consultas brindadas por paciente en lugar de emplear indicadores de resultado propiamente dicho. Los autores de esta entrega consideraron pertinente el empleo de indicadores de resultados en lugar de los de procesos en tanto la situación de salud de la población es una responsabilidad reconocida legalmente por el Estado cubano, por lo que los recursos se asignan con el fin de lograr una modificación positiva de la eficiencia. Una vez identificadas las mejores unidades se podrá profundizar en cuáles son los procesos que esas unidades implementan con vista a que puedan ser generalizados y/o adaptados para el resto de las entidades. La propuesta final de indicadores de resultados a la que se arribó en este estudio refleja el trabajo con los factores de riesgo de las enfermedades bucales, así como las acciones que realizan los estomatólogos desde la pesquisa de enfermedades bucales hasta devolver, mediante tratamientos estomatológicos la salud bucal a una comunidad. La media de eficiencia encontrada así como el número unidades eficientes son comparables con los estudios reportados por Grytten J, Buck D, Widsrom E y otros. ${ }^{5,9-11}$ En dichos estudios se encontró que alrededor del $70 \%$ de las unidades resultaba ser eficientes.

En relación a la categoría docente de la clínica y su efecto sobre la eficiencia, si bien este estudio no arrojó evidencias estadísticas a punto de partida de los resultados debe prestarse atención a los riesgos que para la eficiencia podría tener la incorporación de la actividad docente en las clínicas estomatológicas, con la cual cabría esperar un incremento del gasto de recursos no siempre acompañado de mejores resultados. Por una parte, el gasto por salario de estas clínicas debe ser mayor pues la categorización de sus profesionales trae aparejado un incremento salarial y por la otra, es de esperar que el estudiante ocasione un gasto mayor de materiales, derivado del propio proceso de enseñanza que no siempre se logra compensar con el aporte en términos de los resultados que aquí se midieron. Dado que la política del país va dirigida a extender la enseñanza a la mayor cantidad de clínicas estomatológicas posible, para futuros estudios será necesario incluir un indicador de resultados propio de la actividad docente de manera tal que se logre una valoración más integral de la unidad.

Las clínicas estomatológicas de la provincia Matanzas en el 2006 lograron altos niveles de eficiencia. No obstante para aquellas unidades que no lograron la mejor relación recurso-resultados, estos pueden alcanzarse a través de una estrategia de servicios estomatológicos oportunos y de calidad que generen un incremento del porcentaje de los pacientes que asisten a la clínica que no requieren tratamiento combinado con la reducción del gasto en medicamentos.

\section{REFERENCI AS BI BLI OGRÁFI CAS}

1. Chiarotti Boero S. El derecho humano a la Salud [sitio en Internet]. [citado 12 febrero 2007]. Disponible en: http://www.cajpe.org.pe/rij/bases/doctrina/clad1.htm

2. Palmer S, Torgerson DJ. Definitions of efficiency. BMJ . 1999; 318: 1136.

3. Cosme J, Cárdenas J, Miyar R. Economía y salud conceptos, retos y estrategias. La Habana: OPS/OMS; 1999. [Serie desarrollo de la representación OPS/OMS Cuba No.22-1999]. 
4. Pérez Olivares SA, Gutiérrez Salazar, Soto Cantero L, Vallejos Sánchez A, Casanova Rosado J. Caries dental en primeros molares permanentes y factores socio económicos en escolares de Campeche, México. Rev Cubana Estomatol [serie en Internet]. 2002 [citado 6 Oct 2006]; 39 (3). Disponible en:

http://scielo.sld.cu/scielo.php?script=sci_arttext\&pid=S0034 $\underline{75072002000300001 \& \operatorname{lng}=\mathrm{es} \& \mathrm{n} r \mathrm{~m}=\mathrm{iso}}$

5. Menéndez Laria A. Las tecnologías apropiadas de la salud bucal: un enfoque diferente. Rev Cubana Estomatol [serie en Internet]. 2006[citado 6 Ene 2007]; 43(3). Disponible en:

http://bvs.sld.cu/revistas/est/vol43_3_06/est09306. htm

6. Song F, O'Meara S, Wilson, P, Golder SY, Kleijnen J. The effectiveness and costeffectiveness of prophylactic removal of wisdom teeth. Health Technol Assessment. 2000; 4: 15-25.

7. Beazoglou T, Eklund S, Heffley D, Meiers J, Brown LJ, Bailit H. Economic impact of regulating the use of amalgam restorations. Public Health Rep. 2007; 122:65763.

8. Jarvinen S. Widstrom E. Determinants of costs of orthodontic treatment in the Finís public health service. Swed Dent J. 2004;26 41-9.

9. Buck D. The efficiency of the community dental service in England: a data envelopment analysis. Community Dent Oral Epidemiol. 2004;28:274-80.

10. Grytten J, Ronggen G. Efficiency in provision of public dental services in Norway. Community Dent Oral Epidemiol. 2004;28: 170-6.

11. Widsrom E, Linna M, Niskanen T. Productive efficiency and determinants in the Finnish Public Dental Service. Community Dent Oral Epidemiol. 2004; 32: 31-40.

12. Kontodimopoulos N, Moschovakis G, Aletras VH, Niakas D. The effect of environmental factors on technical and scale efficiency of primary health care providers in Greece. Cost Eff Resour Alloc. 2007; 17: 14-24.

13. Sosa Rosales M, Mojaiber de la Peña A, González Ramos R, Gil Ojeda E. Programa Nacional de Estomatología Integral a la Población. [sitio en Internet]. 2002 [citado 6 Oct 2006]. Disponible en:

http://aps.sld.cu/bvs/materiales/programa/otros/estomatologia/programanacional. pdf

14. Gálvez AM. La aplicación de la economía al campo de la salud, un reto permanente. La Habana: Escuela Nacional de Salud Pública;2006. [Módulo Economía y Salud. Cátedra de Economía de la Salud. Maestría de Economía de la Salud].

15. World Health Organization. The World Health Report 2002. Reducing risks, promoting healthy life. Geneva: World Health Organization; 2002.

16. Albander JM, Brown LJ, Löe H. Clinical features of early-onset periodontitis. J Amer Dental Assoc. 1997; 128: 1393-9.

17. World Health Organization. Global strategies to reduce the health care burden of craniofacial anomalies. Geneva: World Health Organization; 2002. 
18. Avkiran NK. Productivity analysis in the service sector with data envelopment analysis. 2nd ed. Queesland, Australia: University of Queesland;2002.

19. Renner A, Kirigia J M, Zere EA, Barry SP, Kirigia DG, Cand Muthuri K. L.H.K.technical efficiency of peripheral health units in Pujehun district of Sierra Leone: a DEA application. BMC Health Services Res. 2005; 5: 77-87.

20. Banker RD, Charners A, Cooper WW. Some models for estimating technical and sacle inefficiencies in data envelopment analysis management. Science. 1984; 30: 1078-92.

21. Charnes A, Cooper WW, Rhodes E. Measuring the efficiency of decision making units. Eur J Operations Res. 1978;2:429-44.

22. Aletras V, Kontodimopoulos N, Zagouldoudis A, Niakas D. The short-term effect on technical and scale efficiency of establishing regional health systems and general management in Greek NHS hospitals. Health Policy. 2007; 83:3236-45.

Recibido: 25 de mayo de 2008.

Aprobado: 22 de octubre de 2008.

Anaí García Fariñas. Instituto de Farmacia y Alimentos. Universidad de La Habana. La Habana, Cuba.

E-mail:_anaigf@yahoo.es 